\title{
An explicit/implicit Runge-Kutta-based PFEM model for the simulation of thermally coupled incompressible flows
}

\author{
Julio Marti ${ }^{1,2} \cdot$ Pavel Ryzhakov ${ }^{1}$ \\ Received: 8 December 2018 / Revised: 5 February 2019 / Accepted: 11 February 2019 / Published online: 19 February 2019 \\ (c) OWZ 2019
}

\begin{abstract}
A semi-explicit Lagrangian scheme for the simulation of thermally coupled incompressible flow problems is presented. The model relies on combining an explicit multi-step solver for the momentum equation with an implicit heat equation solver. Computational cost of the model is reduced via application of an efficient strategy adopted for the solution of momentum/continuity system by the authors in their previous work. The applicability of the method to solving thermo-mechanical problems is studied via various numerical examples.
\end{abstract}

Keywords Navier-Stokes · thermo-mechanical · Particle finite element method · Lagrangian · Explicit · Benchmark · Boussinesq

\section{Introduction}

Lagrangian finite element models for the simulation of flow problems originated from the ideas presented in [32,33] and [30]. Unlike their fixed grid counterparts, Lagrangian fluid models naturally track the evolving boundaries and do not suffer numerical diffusion. Lagrangian fluid approaches have been further developed for free surface flows in [27] and [18] resulting in what is now generally known as the "particle finite element method" (PFEM), a methodology that combines the features of the classical Lagrangian finite element methods and the mesh-free approaches. The PFEMs have been further advanced by various groups and applied to flows with multi-fluids [16,17], fluid-structure interactions $[15,34,42]$ and multi-fluid-structure interaction problems [38].

Another area where Lagrangian fluid models may be suitable is the thermally coupled flow problems. In such problems, PFEMs facilitate convection of the material properties (which, in case of being temperature dependent, requires solving additional transport equations in case of using fixed

Julio Marti

julio.marti@cimne.upc.edu

1 Centre Internacional de Mètodes Numèrics en Enginyeria (CIMNE), Gran Capitán s/n, 08034 Barcelona, Spain

2 Department of Civil and Environmental Engineering, Universitat Politècnica de Catalunya (UPC), Barcelona, Spain grid approaches). This explains why PFEM-based models were successfully used for analyzing polymer melting [21, 28]. PFEM-based models also appear to be suitable for the simulation of material forming processes (e.g., mold filling), also characterized by a strongly coupled thermo-mechanical nature. Since convection is resolved automatically due to Lagrangian nature of the fluid solver, the heat solver in these models involves solely the diffusion (or diffusion-radiation) equation [23].

In [25], a Lagrangian-based thermally coupled model is applied to the simulation of metal casting. In [3], a basic strategy for the solution of incompressible fluids with thermal convection and free surfaces using the PFEM is presented. The integration scheme is fully implicit; pressure and velocity are uncoupled via the fractional step approach. Besides, the thermal buoyancy effects are considered by introducing the Boussinesq approximation. This strategy is applied to mold filling in [29]. Similar Lagrangian thermally coupled approach is used in commercial software POLYFLOW [1] and applied to glass forming simulation in [13]. A PFEMbased model applied to bottle forming can be found in $[35,40]$.

All the aforementioned PFEM-based approaches rely on fully implicit time integration schemes. Unfortunately, this usually results in very high computational costs in case of mesh degradation and thus strongly limits the application of the PFEM-based model to real-life problems. As shown in [37], time step restriction cannot be eliminated even by a 
frequent re-meshing. The possibility of element inversion at any nonlinear iteration step leads to an immediate failure of the implicit solver [37]. Thus, all the mentioned approaches require estimating a critical time step in order to ensure that no element gets inverted. In practice, this often introduces a prohibitively small time step restriction. Taking into account that the problem solution at each time step involves nonlinear iterations and each nonlinear iteration, in turn, requires solving large linear systems, the resulting methods turn out to have a very large computational cost.

In order to alleviate the above-mentioned drawbacks of the iterative procedure involved in an implicit solution, Idelsohn et al. [14] proposed a scheme, where particles were moved only once, prior to implicit solution of the NavierStokes equations (which was considered linear). Such mesh movement step was fully explicit. The obtained configuration was considered to be the end of step configuration and was not further updated. In order to ensure improved accuracy of this prediction, the particle positions were obtained following the streamline corresponding to velocity at the known time step. The advantage of this method was that it allowed using large time steps without falling into the danger of element inversion as the mesh was considered fixed
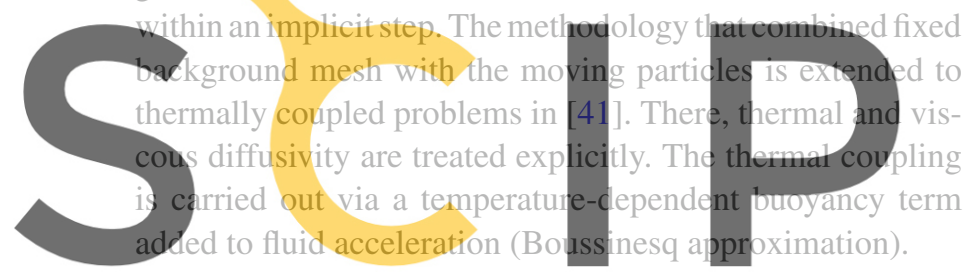

An alternative explicit-implicit Lagrangian method was proposed in [22]. There, domain configuration was accu-

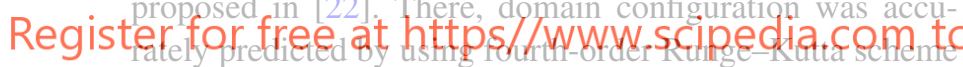

for integrating both the velocity and the particle positions.

Due to the implicit nature of the pressure in incompressible flows, pressure was integrated implicitly. The approach introduced re-meshing prior to pressure solution step, ensuring that no element may be inverted during the implicit step. This allowed to alleviate time step restrictions and eliminate its dependence on the mesh deformation. The computational cost of the method was strongly reduced also due to a technique that allowed solving for the pressure only once per time step.

In the present work, the explicit-implicit scheme proposed in [22] is extended to thermally coupled problems (a fixed grid version of the scheme can be consulted in [36] and [39] ). For the problems where buoyancy effects are essential, Boussinesq term is added to the fluid acceleration.

The paper is organized as follows: Sect. 2 presents the governing equations of a thermally coupled incompressible fluid. The solution algorithm is outlined. Section 3 is devoted to solution of various benchmarks. First, a thermal square cavity is solved and the solution is validated using the bibliographic data. Next, a problem involving a thermally coupled fluid flow in a backward facing step is solved. Ultimately, a thermally coupled fluid sloshing example is simulated. Section 4 is devoted to the summary and concluding remarks.

\section{Numerical model for thermally coupled incompressible flows}

In thermally coupled problems, the energy equation is coupled to the momentum/continuity system via the convective velocity. For the low-speed flows with nonnegligible buoyancy effects, Boussinesq hypothesis is often employed, provided that temperature gradients are small. It allows to account for the aforementioned thermal buoyancy phenomena using an incompressible flow model [26]. According to this hypothesis, the buoyant term of the momentum equation is assumed to be temperature dependent, while the density is assumed constant everywhere else in the model. Boussinesq solvers allow solving the energy and the momentum-continuity system in a staggered fashion. The popularity of Boussinesq hypothesis is specially related to its practical applicability and the simplicity of its implementation.

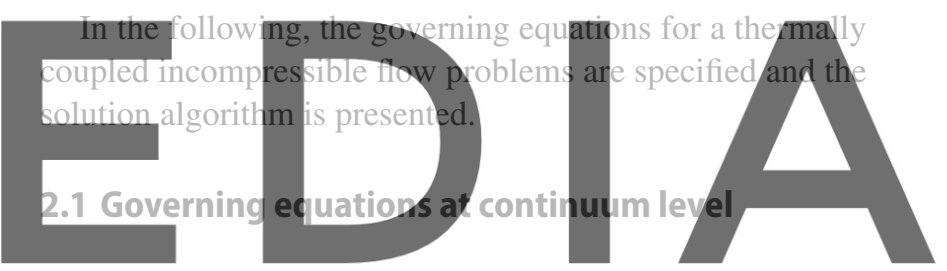

Let $\Omega \subset R^{3}$ be a bounded domain containing viscous incom-

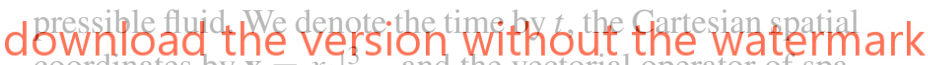
coordinates by $\mathbf{x}=\left.x_{i}\right|_{i=1}$ and the vectorial operator of spatial derivatives by $\nabla=\left\{\partial_{x_{i}}\right\}_{i=1}^{3}$. The evolution of the velocity $\mathbf{v}=\mathbf{v}(\mathbf{x}, t)$, the pressure $p=p(\mathbf{x}, t)$ and the temperature $T=T(\mathbf{x}, t)$ is governed by the following equations:

$$
\begin{aligned}
\rho \frac{\partial \mathbf{v}}{\partial t}+\rho(\mathbf{v} \cdot \nabla) \mathbf{v}-\nabla \cdot(\mu \mathbf{D})+\nabla p & =\mathbf{f}, \\
\nabla \cdot \mathbf{v} & =0 \\
\rho C \frac{\partial T}{\partial t}+\rho C \mathbf{v} \cdot \nabla T-\kappa \Delta T & =0 .
\end{aligned}
$$

where $\mu$ is the fluid dynamic viscosity, $\rho$ is the density, $p$ is the fluid pressure, $\mathbf{\mathbf { D }}=\frac{1}{2}\left(\nabla \mathbf{v}+\nabla^{T} \mathbf{v}\right)$ is the symmetric part of the velocity gradient tensor, $C$ is the heat capacity and $\kappa$ is the thermal conductivity. According to Boussinesq hypothesis, the body force $\mathbf{f}$ is computed as

$\mathbf{f}=\rho \mathbf{g}\left[1-\beta\left(T-T_{0}\right)\right]$

where $\mathbf{g}$ is the gravity acceleration, $T$ and $T_{0}$ are the actual temperature and the reference temperature, respectively, and $\beta$ is the thermal diffusion coefficient. 


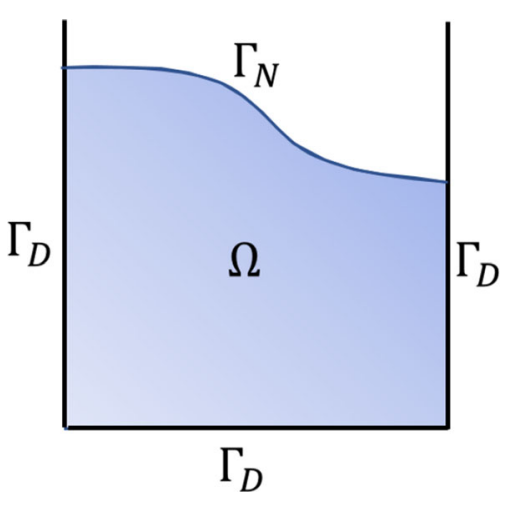

Fig. 1 Domain and boundaries

The position $\mathbf{x}$ of a given material particle (which in PFEM coincides with the mesh node once the problem is discretized) is given by:

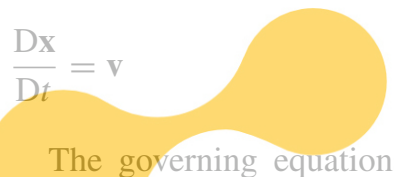

The governing equations are completed with standard Dirichlet and Neumann boundary conditions on $\Gamma_{D}$ and $\Gamma_{N}$, respectively. The domain boundaries are shown in Fig. 1.
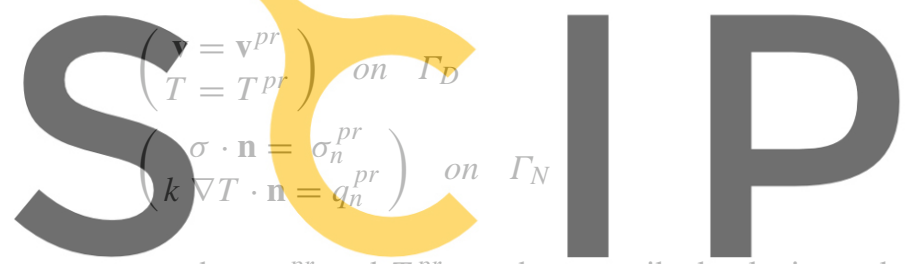

(6)

where $\mathbf{v}^{p r}$ and $T^{p r}$ are the prescribed velocity and temperature, respectively, $\mathbf{n}$ is the outer unit normal to $\Gamma_{N}, \sigma_{n} \mathrm{pr}$

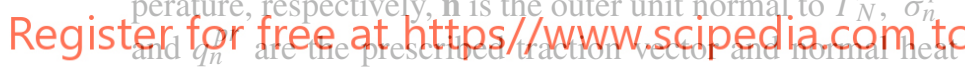
flux. Note that $\sigma$ stands for Cauchy stress tensor.

The discrete version of the governing equations is presented next.

\subsection{Governing equations at discrete level}

In the present work, we use a semi-explicit solver of [22] for the mechanical problem and a Backward Euler scheme for the heat equation. An updated Lagrangian reference frame is considered. The semi-explicit solver for the momentum/continuity/position system is based on the fourth-order Runge-Kutta scheme and is characterized by the secondorder temporal accuracy.

Considering linear finite element approximations for the temperature (note that $\mathbf{N}$ is the vector of shape functions)

$$
T(\mathbf{x})=\mathbf{N}^{T}(\mathbf{x}) \bar{T}
$$

heat equation discretized in space and time reads (note the absence of the convective term due to adopting the Lagrangian reference frame) $\rho C \mathbf{M} \frac{\bar{T}^{n+1}}{\Delta t}+\kappa \mathbf{L} \bar{T}^{n+1}=\rho C \mathbf{M} \frac{\bar{T}^{n}}{\Delta t}$.

A linear approximation of the same order is used for the velocity and the pressure

$p(\mathbf{x})=\mathbf{N}^{T}(\mathbf{x}) \bar{p}$

$\mathrm{v}_{i}(\mathbf{x})=\mathbf{N}^{T}(\mathbf{x}) \overline{\mathrm{v}}_{i}$

We note that the nodal variables are distinguished from their continuum counterparts by an overbar.

Applying the above spatial approximations and the fourthorder Runge-Kutta time integration scheme, the following equations are obtained (see [22] for further details):

$$
\begin{aligned}
\overline{\mathbf{v}}^{n+1}= & \overline{\mathbf{v}}^{n}+\frac{\Delta t}{6} \mathbf{M}^{-1}\left(\mathbf{r}_{1}+2 \mathbf{r}_{2}+2 \mathbf{r}_{3}+\mathbf{r}_{4}\right) \\
& -\frac{\Delta t}{6 \rho} \mathbf{M}^{-1} \mathbf{G} \bar{p}^{n+1}
\end{aligned}
$$

where intermediate residuals, nodal velocities and positions are defined as:
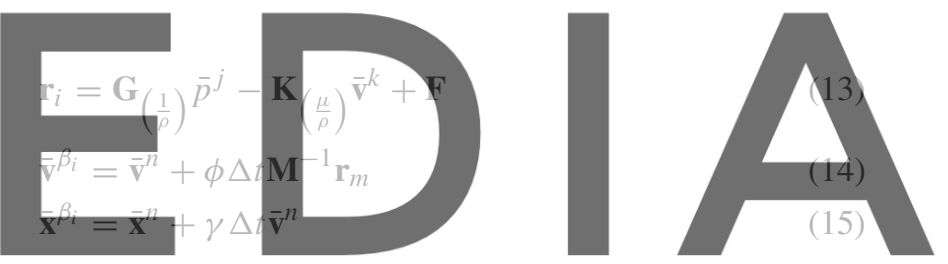

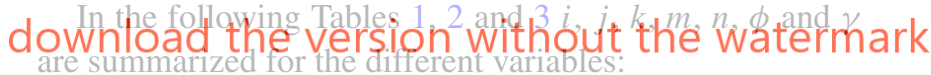 \\ Introducing an intermediate velocity $\tilde{\mathbf{V}}$, following the frac-} tional step approach [4] for decoupling the velocity and the pressure, Eq. (12) can be replaced by the following three equations to be solved sequentially:

Table 1 Intermediate residuals

\begin{tabular}{lll}
\hline $\mathbf{r}_{i}$ & & \\
\hline$i$ & $j$ & $k$ \\
\hline 1 & $n$ & $n$ \\
2 & $n$ & $\beta_{1}$ \\
3 & $\beta_{2}$ & $\beta_{2}$ \\
4 & $\beta_{3}$ & $\beta_{3}$ \\
\hline
\end{tabular}

Table 2 Velocities

\begin{tabular}{lll}
\hline$\overline{\mathbf{v}}^{\beta_{i}}$ & & \\
\hline$i$ & $\phi$ & $m$ \\
\hline 1 & $1 / 2$ & 1 \\
2 & $1 / 2$ & 2 \\
3 & 1 & 3 \\
\hline
\end{tabular}


Table 3 Nodal positions

\begin{tabular}{lll}
\hline$\overline{\mathbf{x}}^{\beta_{i}}$ & & \\
\hline$i$ & $\gamma$ & $n$ \\
\hline 1 & $1 / 2$ & $\mathrm{n}$ \\
2 & $1 / 2$ & $\beta_{1}$ \\
3 & 1 & $\beta_{2}$ \\
\hline
\end{tabular}

$\tilde{\mathbf{v}}=\overline{\mathbf{v}}^{n}+\frac{\Delta t}{6} \mathbf{M}^{-1}\left(\mathbf{r}_{1}+2 \mathbf{r}_{2}+2 \mathbf{r}_{3}+\mathbf{r}_{4}\right)$

$+\frac{\Delta t}{6} \mathbf{M}^{-1} \mathbf{G}_{\left(\frac{1}{\rho}\right)} \bar{p}^{n}$

$\frac{\Delta t}{6} \mathbf{L} \bar{p}^{n+1}=\frac{\Delta t}{6} \mathbf{L} \bar{p}^{n}-\mathbf{D}_{\rho} \tilde{\mathbf{v}}$

$\overline{\mathbf{v}}^{n+1}=\tilde{\mathbf{v}}+\frac{\Delta t}{6} \mathbf{M}^{-1} \mathbf{G}_{\left(\frac{1}{\rho}\right)}\left(\bar{p}^{n+1}-\bar{p}^{n}\right)$

Particle's position $\overline{\mathbf{x}}$ at time $n+1$ can be found as

$\overline{\mathbf{x}}^{n+1}=\overline{\mathbf{x}}^{n}+\frac{\Delta t}{6}\left(\overline{\mathbf{v}}^{n}+2 \overline{\mathbf{v}}^{\beta 1}+2 \overline{\mathbf{v}}^{\beta 2}+\overline{\mathbf{v}}^{\beta 3}\right)$

$$
\begin{aligned}
& \mathbf{D}_{\rho}=\sum_{\text {elem }} \int_{\Omega} \rho \mathbf{N} \nabla \mathbf{N}^{T} d \Omega \\
& \mathbf{L}=\sum_{\text {elem }} \int_{\Omega} \nabla \mathbf{N}^{T} \nabla \mathbf{N} d \Omega
\end{aligned}
$$

Equation (17) must be stabilized to avoid numerical oscillation due to the equal order of approximation for velocity and pressure. The stabilization technique used in the present work is the algebraic sub-grid scale method [5]. For the sake of simplicity, stabilization terms are omitted here. They can be consulted in [22] where the mechanical solver was derived.

\subsection{Solution algorithm}

The problem to be solved can be formulated as: given the nodal positions, the velocity, the pressure and the temperature at time step $t^{n}$, find these variables at $t^{n+1}$. The overall solution strategy according to the method proposed is summarized in Table 4.

The matrices in the above equations are defined as:
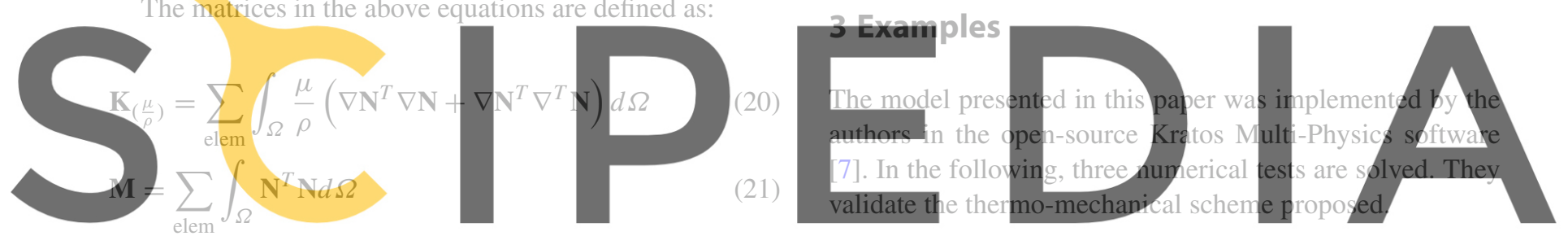

Register for free at $\mathrm{N}^{T}$ https//www.scipedia.com to

$\mathrm{G}_{\left(\frac{1}{\rho}\right)}=\sum_{\mathrm{elem}} \int_{\Omega} \frac{1}{\rho} \nabla \mathrm{N}^{T} \mathrm{~N} d \Omega$

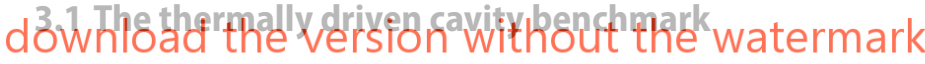

This test models the fluid flow in two-dimensional unitary square. Different temperatures are prescribed and maintained

Table 4 Lagrangian explicit-implicit solution algorithm for thermally coupled incompressible flow problems

1. Knowing the velocity $\mathbf{v}$, pressure $p$, temperature $T$ and nodal position $\mathbf{x}$ corresponding to time $t^{n}$ perform the explicit step:

- For $i$ from 1 to 4

Evaluate intermediate Runge-Kutta residuals $\mathbf{r}_{i}(i=1, \ldots, 4)$ using Eqs.(13).

Move particles to the new position $\overline{\mathbf{x}}^{\beta_{i}}$ (Eq.(19)).

Update elemental matrices and vectors according to the new nodal positions (Eqs. 20-25).

2. Re-mesh the fluid domain

3. Perform the implicit step: solve the Poisson's equation for the pressure (Eq.(17)). Result: $\bar{p}^{n+1}$.

4. Correct the velocity to obtain a divergence-free solution. Result: $\overline{\mathbf{v}}^{n+1}$ (Eq.(18)).

5. Solve the heat equation (Eq. (9)). Result: $\bar{T}^{n+1}$

6. Compute the Boussinesq term for the next time step using the obtained temperature (Eq. (4)).

7. Go to the next time step. 


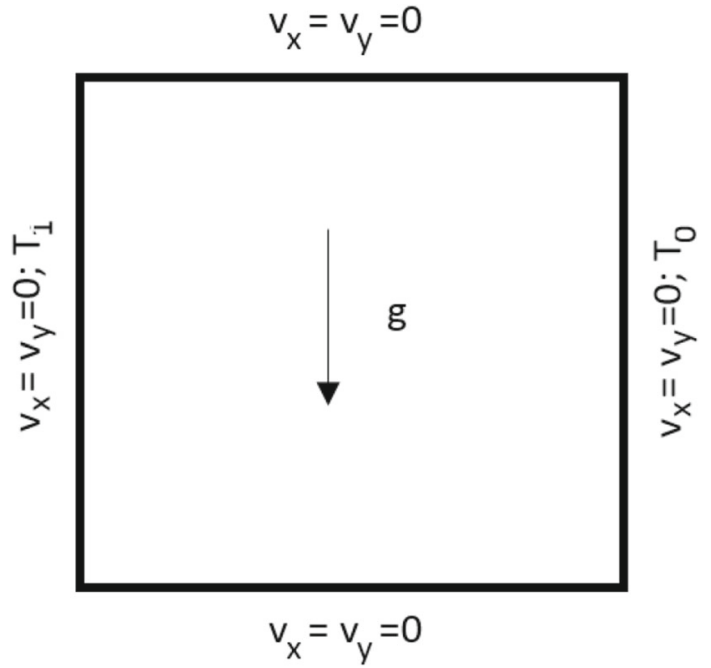

Fig.2 Boundary conditions for thermal cavity benchmark problem

at the vertical walls. Convective transport in this test is known as "natural convection." It develops exclusively due to nonzero temperature gradients. This example is used to test the numerical algorithms designed for the integration of the Navier-Stokes equations in incompressible recnculating flows. Boussinesq approxinnation [2] is valid due to very small temperature variation. The input data provided beld description given in [8]. The insulated (adiabatic condition is considered), velocity at all boundaries is set to zero. Vertical walls' tem-

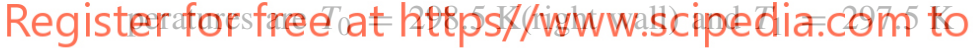
(left wall). Fluid inside the cavity is initially at rest. Its initial temperature equals the mean of the temperatures on the vertical walls. The scheme of the example is shown in Fig. 2.

The simulations were executed for a range of Rayleigh number $\left(R a=\frac{g \beta H^{3} \Delta T}{\alpha \nu}\right)$ values: $R a=10^{6}, R a=10^{5}$ and $R a=10^{4}$. Prandtl number $\left(\operatorname{Pr}=\frac{v}{\alpha}\right)$ was set to $(0.71)$. Note that $g, \beta, \alpha$ and $v$ are the gravity, thermal diffusion coefficient, thermal diffusivity and kinematic viscosity, respectively. A constant temperature difference of $\Delta T=1 \mathrm{~K}$ was applied to the two vertical walls, adjusting the thermal diffusion coefficient $\beta$ until obtaining the desired Rayleigh number, keeping all the other variables constant.
Table 6 Numerical solution for the thermal square cavity, $R a=10^{5}$. Comparison with [8]

\begin{tabular}{llrr}
\hline$R a$ & Data & \multicolumn{1}{c}{ RK } & \multicolumn{1}{c}{ Davis } \\
\hline $10^{5}$ & $v_{x, \max }(x=0.5)$ & 34.924 & 34.870 \\
$10^{5}$ & $y_{\max }$ & 0.862 & 0.855 \\
$10^{5}$ & $v_{y, \max }(y=0.5)$ & 67.910 & 67.910 \\
$10^{5}$ & $x_{\max }$ & 0.0576 & 0.067 \\
\hline
\end{tabular}

The following values were used for the main variables

$$
\begin{aligned}
& \rho=1 \frac{\mathrm{kg}}{\mathrm{m}^{3}} \\
& v=0.001 \frac{\mathrm{m}^{2}}{\mathrm{~s}} \\
& g_{y}=-10 \frac{\mathrm{m}}{\mathrm{s}^{2}} \\
& \alpha=0.001 \frac{\mathrm{m}^{2}}{\mathrm{~s}}
\end{aligned}
$$

The simulations were carried out using mesh size $h=$

to

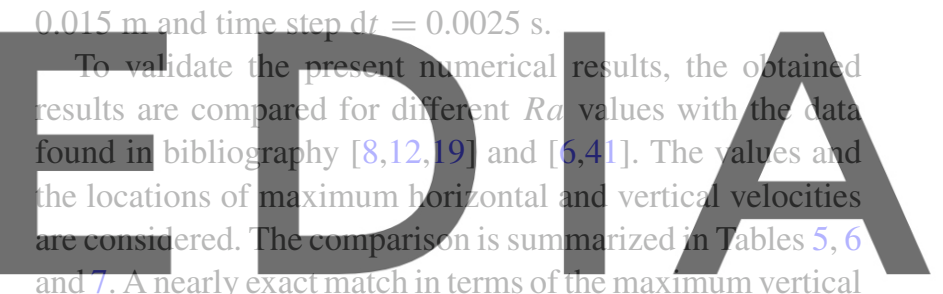

and 7. A nearly exact match in terms of the maximum vertica and horizontal velocity for the entire range of the considered

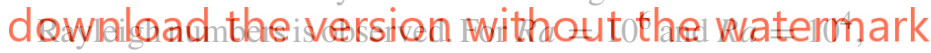
the results are compared against the fixed grid simulations of $[6,8]$ and the combined PFEM-Eulerian simulation of [41]. For $R a=10^{5}$, no results are presented in [6] and [41]; thus, the comparison is made only with [8]. One can see a very good agreement among the results. The largest discrepancy is observed in the location of the maximum vertical velocity. One can see that for $R a=10^{6}$ the difference between the compared values is as high as $20 \%$. For smaller Rayleigh number, this discrepancy diminishes.

Results shown in Fig. 3 compare the isotherms obtained in our simulations with those corresponding to the reference solution [9]. The results are in very good agreement with the benchmark solution.
Table 5 Numerical solution for the thermal square cavity, $R a=10^{6}$. Comparison with $[6,8],[41]$

\begin{tabular}{llccrr}
\hline$R a$ & Data & RK & Davis [8] & Corzo [6] & Sklar [41] \\
\hline $10^{6}$ & $v_{x, \max }(x=0.5)$ & 65.45 & 65.81 & 64.558 & 64.483 \\
$10^{6}$ & $y_{\max }$ & 0.85 & 0.852 & 0.851 & 0.845 \\
$10^{6}$ & $v_{y, \max }(y=0.5)$ & 213.07 & 214.64 & 221.572 & 218.054 \\
$10^{6}$ & $x_{\max }$ & 0.0474 & 0.0396 & 0.067 & 0.037 \\
\hline
\end{tabular}


Table 7 Numerical solution for the thermal square cavity, $R a=10^{4}$. Comparison with $[6,8,41]$

\begin{tabular}{|c|c|c|c|c|c|}
\hline$R a$ & Data & RK & Davis [8] & Corzo [6] & Sklar [41] \\
\hline $10^{4}$ & $v_{x, \max }(x=0.5)$ & 16.250 & 16.182 & 16.282 & 15.982 \\
\hline $10^{4}$ & $y_{\max }$ & 0.821 & 0.823 & 0.822 & 0.824 \\
\hline $10^{4}$ & $v_{y, \max }(y=0.5)$ & 19.541 & 19.509 & 19.547 & 19.378 \\
\hline $10^{4}$ & $x_{\max }$ & 0.115 & 0.120 & 0.123 & 0.116 \\
\hline
\end{tabular}

Figure 4 shows the horizontal velocity profiles in the cavity along the vertical line at $x=0.5$. One can see that the present results coincide with those of [6] for all the considered values of Rayleigh number.

The results of the present method are compared against those of the former version of thermally coupled purely Lagrangian implicit PFEM model in Fig. 5. Rayleigh number considered here is $R a=10^{6}$, and nine isotherms are shown so as to facilitate the comparison with the reference results. Figure 5a shows the solution obtained in the present work, while Fig. 5b and c displays the isotherms of the reference solution [8] and the PFEM model of [24], respectively. One can see that all the isotherms in the upper part of the graphs are nearly identical for all the models, while the third isotherm (counted from the bottom) obtained by the present approach

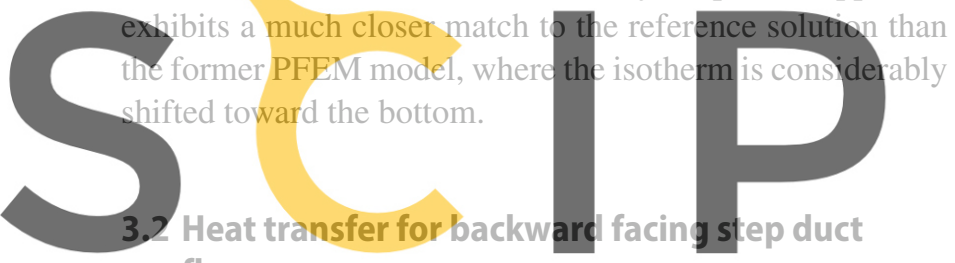
flows

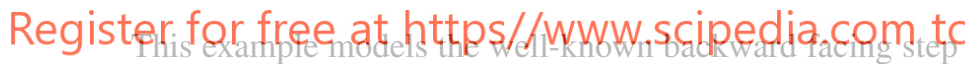
duct flow, which is one of the very commonly simulated CFD problems. Although the geometry of the test is simple, it is characterized by complex flow physics. This example is commonly used to validate conjugate heat transfer models [31]. In our case for the sake of simplicity, heat transfer is modeled only in a single homogeneous fluid.

Geometrical details, boundary and initial conditions of the problem are depicted in Fig. 6. The problem was simulated for two values of Reynolds number: $R e=100$ and $R e=$ 800. Prandtl number was maintained identical in both cases: $\operatorname{Pr}=0.71$. Fluid conductivity was set as $\kappa=0.02 \mathrm{~W} /(\mathrm{m}$. $\mathrm{K})$. Gravity is neglected.

Obtained velocity and temperature fields are shown in Figs. 7, 8, 9 and 10. The results obtained using the present semi-explicit model are compared with those of an implicit fixed grid model. The latter solver was validated and applied to the thermally coupled backward facing step in [20].

The correlation between the solutions obtained for $R e=$ 100 using the present model and the Eulerian model is shown in Figs. 7 (velocity) and 8 (temperature). The solutions are nearly identical.
For $R e=800$, some differences between the Lagrangian and the Eulerian simulation results manifest. Figures 9 and 10 show the cold fluid entering from the left reattaches on the solid wall at approximately $x=6 \mathrm{~m}$. After the reattachment point, the cold fluid in contact with the relatively hot solid wall is heated. A hot spot that appears in the fluid region extending from $x=0$ to the reattachment point results from the fluid trapped between the entering fluid stream and the channel. However, the posterior development of the thermal boundary layer downstream of the impingement point is slightly different. This is related with the evolution of the velocity that can be observed in detail in Fig. 12 which affects the evolution of the heated layer of fluid. While the Eulerian model exhibits a steady-state solution, the Lagrangian model shows a periodic solution. The difference is particularly evi-

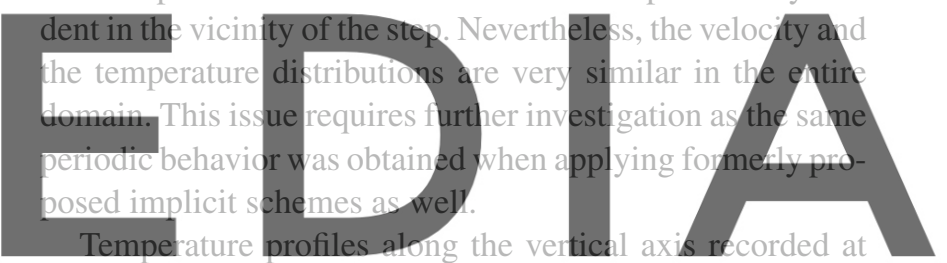
$x=6,14$ and 30 are plotted in Figs. 11 and 12. Once again,

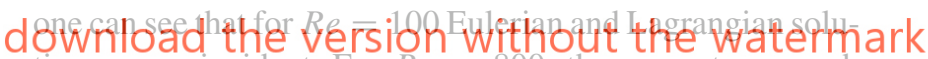
tions are coincident. For $R e=800$, the present approach slightly deviates from the reference solution due to the unsteady nature of the flow in the vicinity of the step. This difference is largest along the horizontal axis of the channel at $y=0.5$. Further away from the step, the solutions completely coincide.

\subsection{Sloshing in a hot container}

While previous examples involved analysis of problems with fixed boundaries and were solved for the sake of the solver validation, the next test deals with a problem that involves a domain with moving boundaries. It deals with a free surface flow in a container with hot walls. Tests of this kind were proposed in [11] for illustrating the capability of thermally coupled PFEM-solvers. The example focuses on the impact of temperature-induced viscosity changes upon the flow behavior.

Domain configuration at $t=0$ is shown in Fig. 13. Noslip boundary condition is prescribed at all the walls of the domain. Free surface (zero tractions) condition is prescribed 
Fig. 3 Natural convection in a square cavity. Temperature field and isolines. Comparison with [9]. Blue and red colors in the present method results

correspond to 297.5 and 298.5

$\mathrm{K}$, respectively

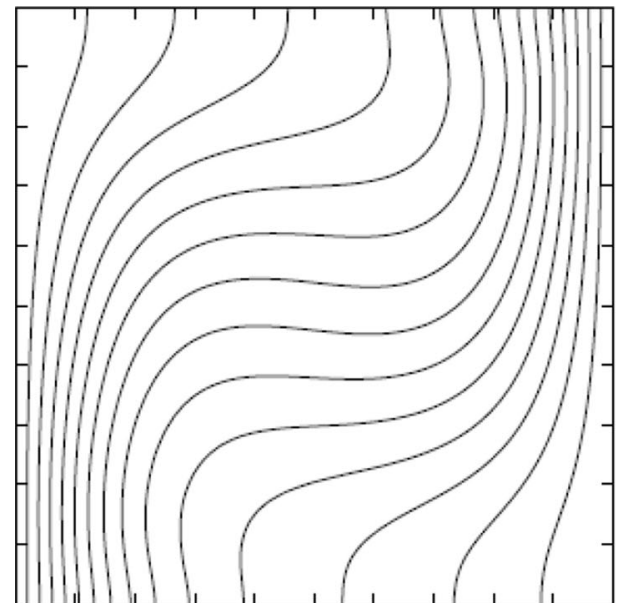

(a) $\mathrm{Ra}=10^{4},[9]$

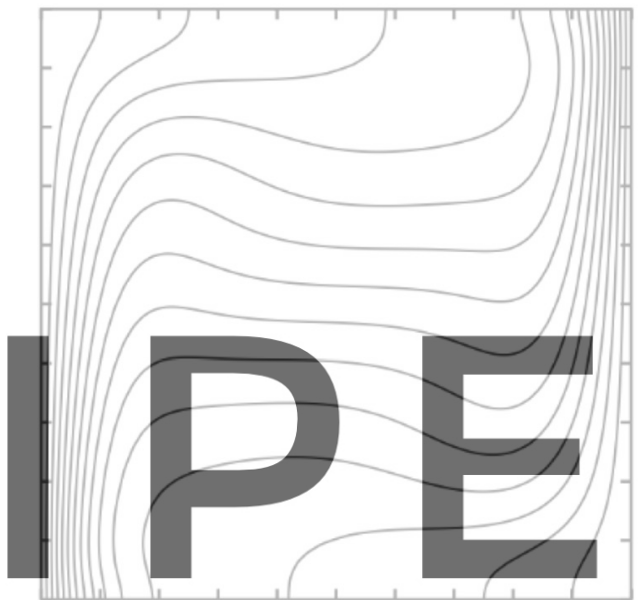

(c) $\mathrm{Ra}=10^{5},[9]$

Register for free at https//www.scipedia.com to download the version without the watermark

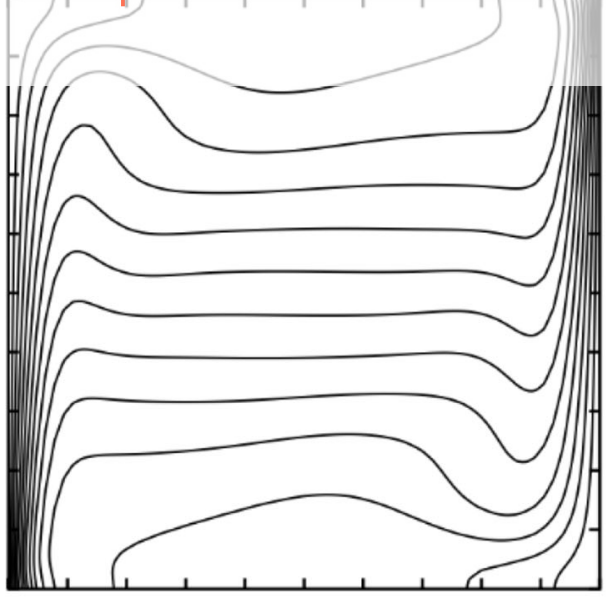

(e) $\mathrm{Ra}=10^{6},[9]$

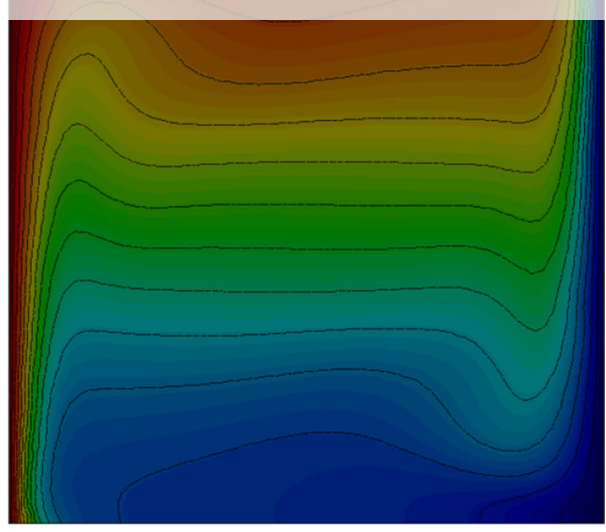

(f) $\mathrm{Ra}=10^{6}$, present work at the free surface. The dimensions of the domain are: $\mathrm{H}=$ $0.1 \mathrm{~m}$ and $\mathrm{h}=0.02 \mathrm{~m}$. The free surface shape is sinusoidal. The properties are: density $\rho=1000 \mathrm{~kg} / \mathrm{m}^{3}$ and gravity $g=-9.8 \mathrm{~m} / \mathrm{s}^{2}$.
The initial temperature of the fluid is set to $T_{0}=273 \mathrm{~K}$. Walls are kept at a fixed temperature: $T_{w}$ (the simulation is carried out for three values of wall temperature: 273,373 and $473 \mathrm{~K}$ ). The viscosity is computed from the temperature using the following expression: 
Fig. 4 Natural convection in a square cavity. Horizontal velocity profiles along the vertical cut at $x=0.5$. Comparison with [6]

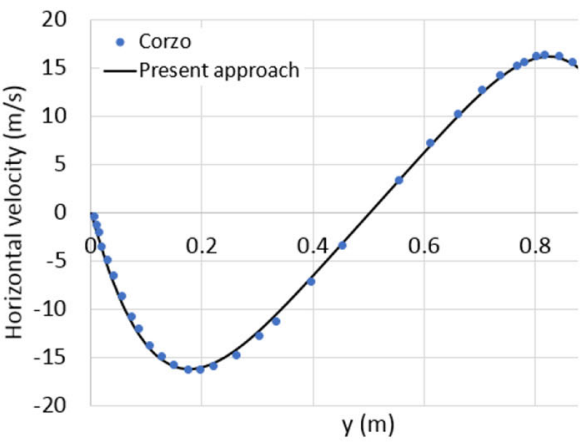

(a) $\mathrm{Ra}=10^{4}$

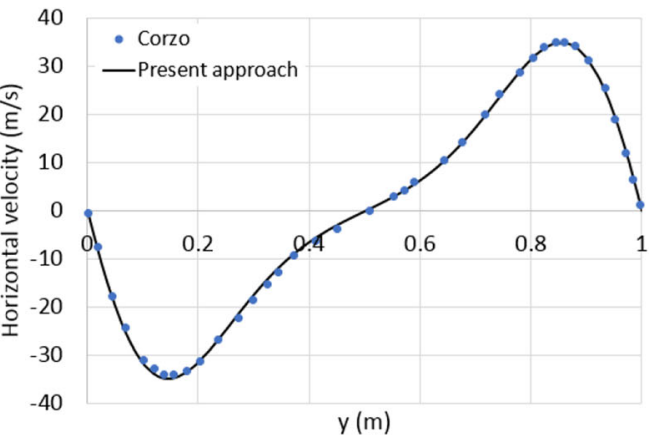

(b) $\mathrm{Ra}=10^{5}$
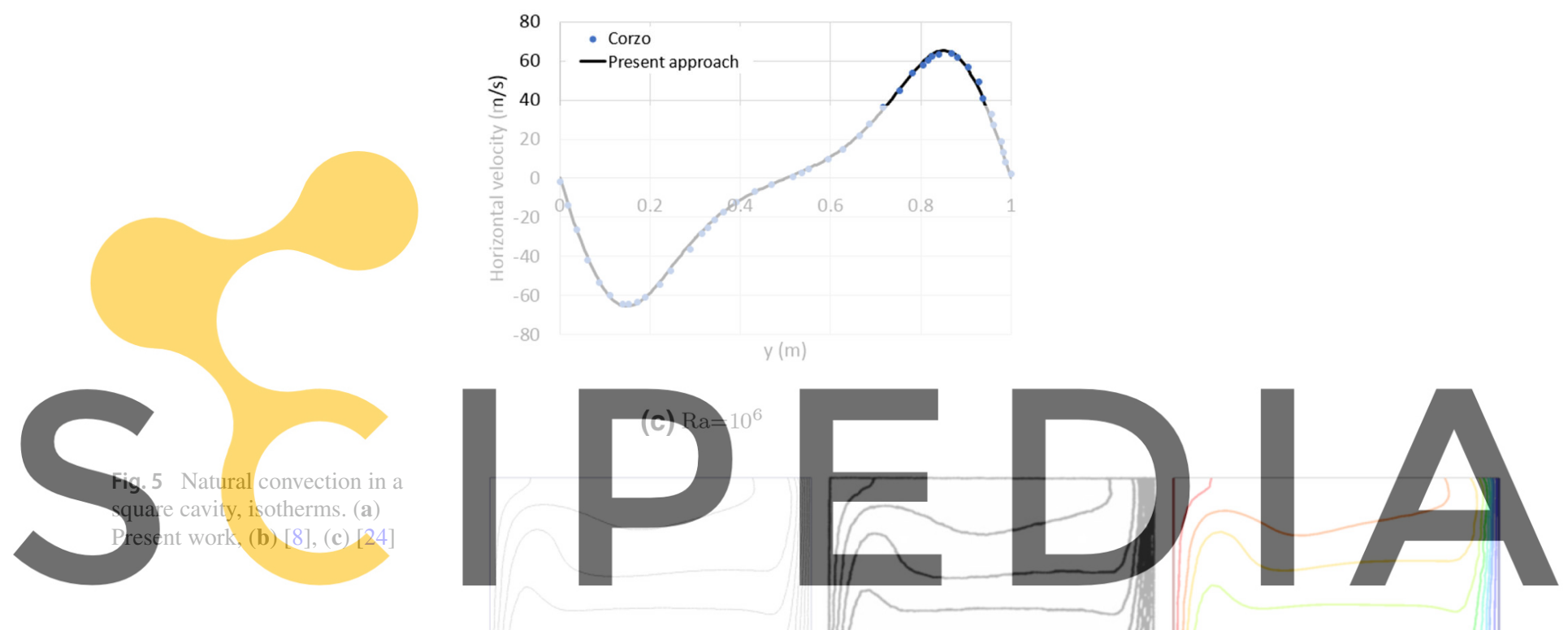

Register for free at https//www.scipedia.com to download the version without the watermark

Fig. 6 Thermally coupled backward facing step.

Geometric details, initial and boundary conditions

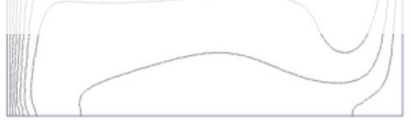

(a)

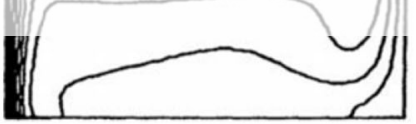

(b)

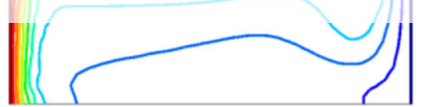

(c)

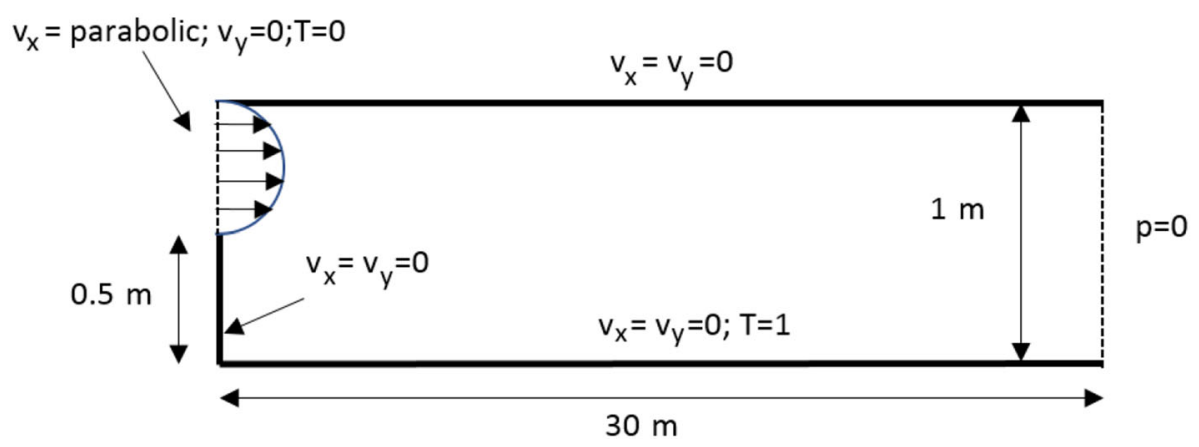




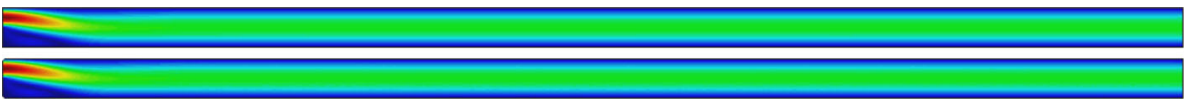

Fig. 7 Thermally coupled backward facing step. Velocity magnitude contours $(R e=100, \operatorname{Pr}=0.71)$ at 40 s. Eulerian (above) versus Lagrangian (below) simulation results. Red and blue colors stand for 1.5 and $0 \mathrm{~m} / \mathrm{s}$, respectively. (Color figure online)

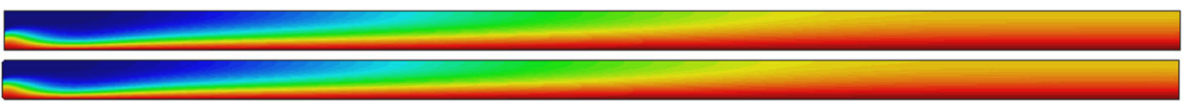

Fig. 8 Thermally coupled backward facing step. Temperature contours $(\operatorname{Re}=100, \operatorname{Pr}=0.71)$ at $40 \mathrm{~s}$. Eulerian (above) versus Lagrangian (below) simulation results. Red and blue colors stand for 0 and $1 \mathrm{C}$, respectively. (Color figure online)

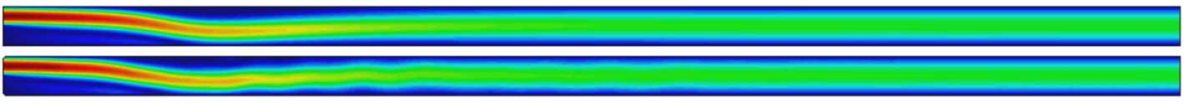

Fig. 9 Thermally coupled backward facing step. Velocity magnitude contours ( $R e=800, \operatorname{Pr}=0.71)$ at $40 \mathrm{~s}$. Eulerian (above) vs Lagrangian (below) simulation results. Red and blue colors stand for 1.5 and $0 \mathrm{~m} / \mathrm{s}$, respectively. (Color figure online)

Fig. 10 Thermally coupled backward facing step. Temperature contours ( $R e=800, \operatorname{Pr}=0.71)$ at $40 \mathrm{~s}$. Eulerian (above) vs Lagrangian (below) simulation results. Red and blue colors stand for 0 and $1 \mathrm{C}$, respectively. (Color figure online)
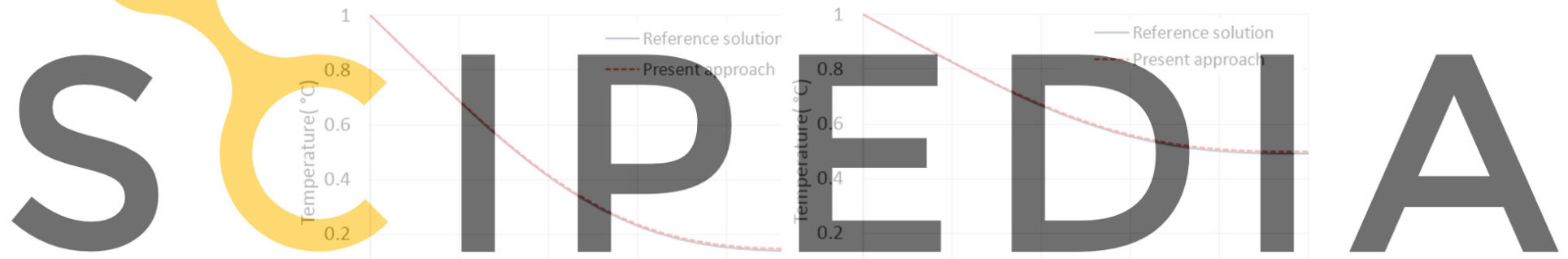

\section{Register for free at https//Wwww.schipedia.coln to download the versión without the watermark}

(a) $\mathrm{x}=6$.

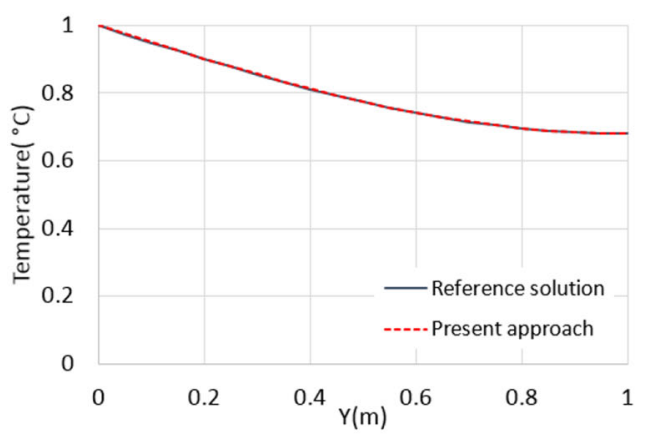

(c) $\mathrm{x}=30$ (b) $\mathrm{x}=14$

Fig. 11 Thermally coupled backward facing step. Comparison of temperature profiles at $x=6$; 14 ; and 30 for the case of $R e=100$. Eulerian (reference) [20] versus Lagrangian (present approach) simulation results 


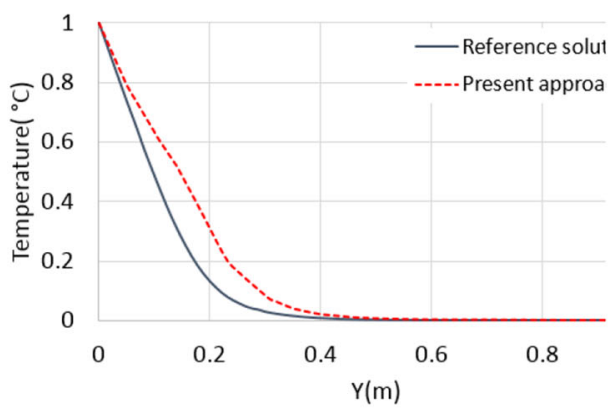

(a) $\mathrm{x}=6$.

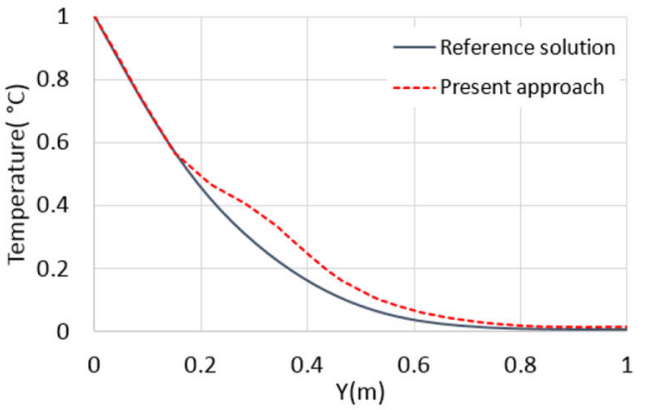

(b) $\mathrm{x}=14$.

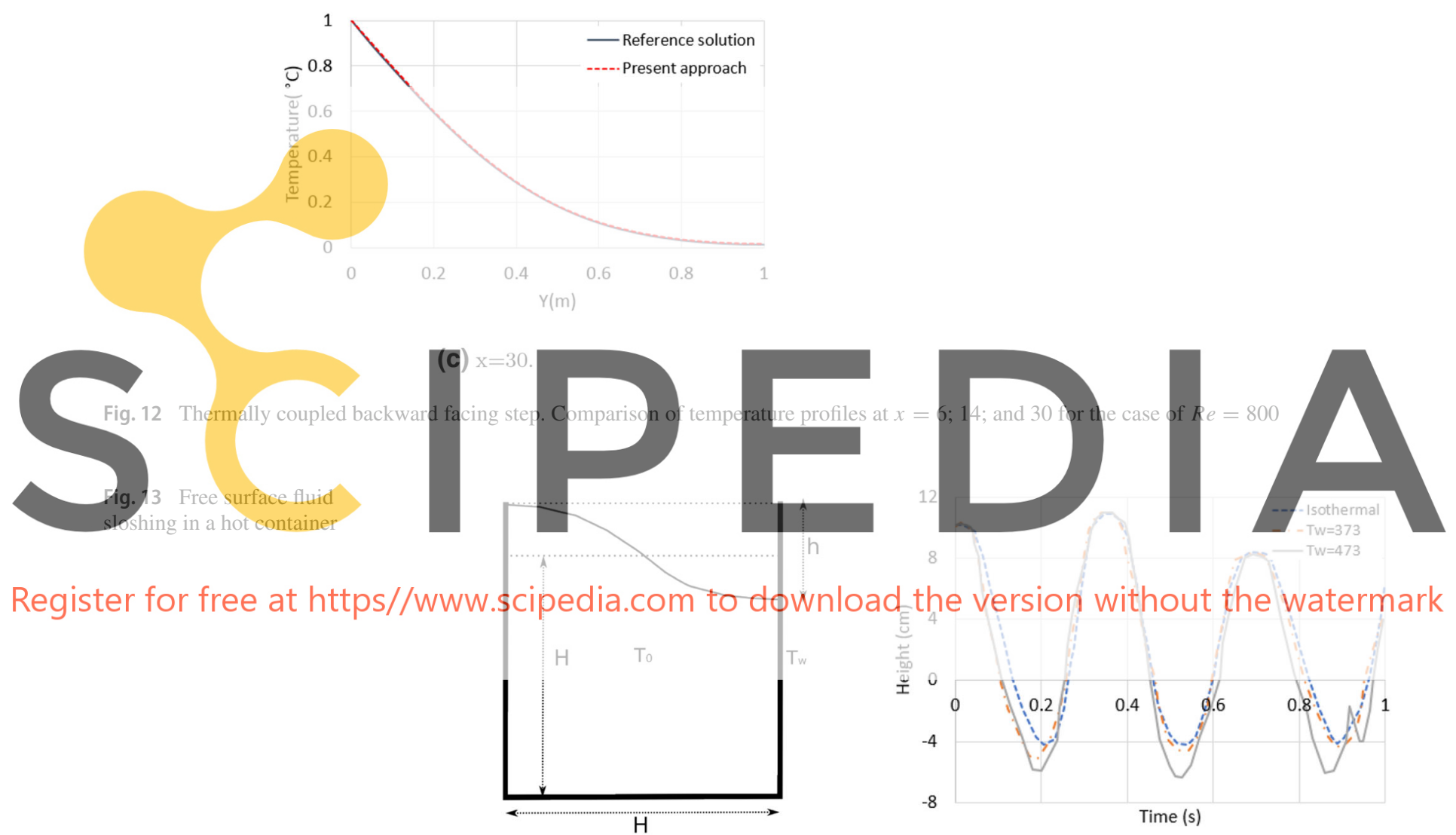

(a) Initial domain geometry

(b) Wave height at the left wall

$\begin{aligned} v= & -2.996 \cdot T^{3} \cdot 10^{-7}+0.000379 \cdot T^{2}-0.161 \cdot T \\ & +23.12\end{aligned}$

The above formula mimics the relative viscosity change of water between 273 and $473 \mathrm{~K}$ [10]. The heat capacity $C$ and the conductivity $\kappa$ of the fluid were set to: $4000.0 \mathrm{~J} /(\mathrm{kg} \cdot \mathrm{K})$ and $2000.0 \mathrm{~W} /(\mathrm{m} \cdot \mathrm{K})$, respectively.

A total time of $1 s$ is simulated. The domain is discretized with an unstructured triangular mesh of size $0.002 \mathrm{~m}(6000$ elements approximately).
We test the impact of the thermal effects upon the fluid behavior considering that the walls of the container are hot. Domain configurations showing temperature distributions are shown at various time instances in Fig. 14. As the temperature propagates (mainly due to diffusion in the present case) from the walls into the fluid volume, the viscosity changes and the differences in the free surface location become more and more evident. In particular, at $t=0.9 \mathrm{~s}$ the case characterized by the highest wall temperature exhibits a significantly different solution, as the temperature increases significantly nearly in the entire domain (except for the small 
Fig. 14 Sloshing in a hot container. Temperature distribution at various time instances

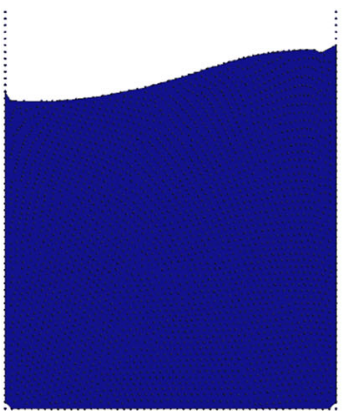

(a) $T_{w}=273 \mathrm{~K}, \mathrm{t}=0.2 \mathrm{~s}$

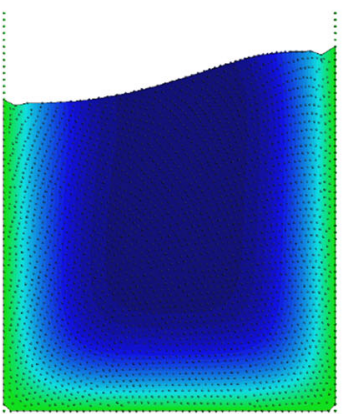

(d) $T_{w}=373 \mathrm{~K}, \mathrm{t}=0.2 \mathrm{~s}$

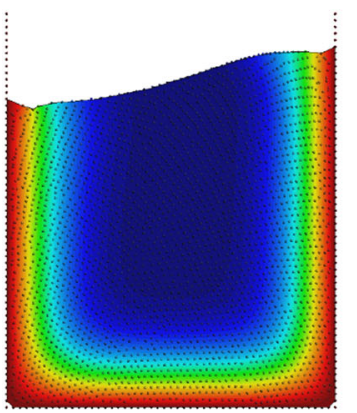

(g) $T_{w}=473 \mathrm{~K}, \mathrm{t}=0.2 \mathrm{~s}$

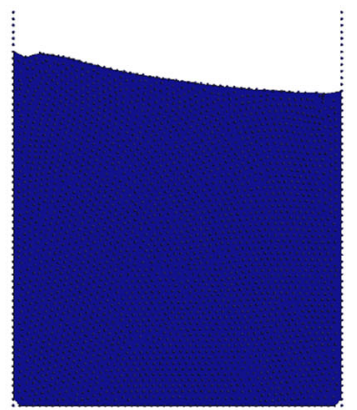

(b) $T_{w}=273 \mathrm{~K}, \mathrm{t}=0.4 \mathrm{~s}$

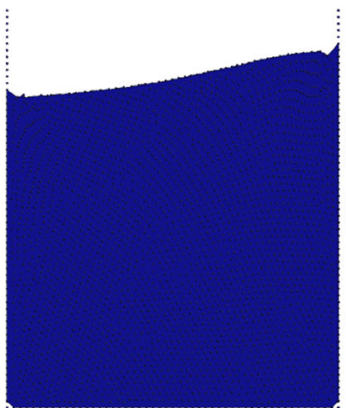

(c) $T_{w}=273 \mathrm{~K}, \mathrm{t}=0.9 \mathrm{~s}$

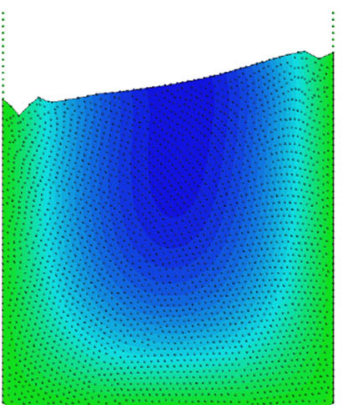

(f) $T_{w}=373 \mathrm{~K}, \mathrm{t}=0.9 \mathrm{~s}$

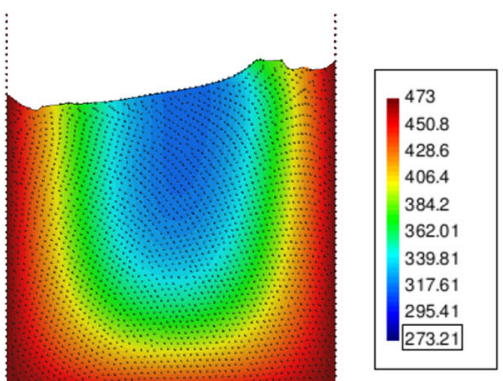

(i) $T_{w}=473 \mathrm{~K}, \mathrm{t}=0.9 \mathrm{~s}$ area in the center). Attaining lower viscosity, the fluid moves faster, therefore, e.g., at $0.9 \mathrm{~s}$, the crest of the wave moves further from the right wall as the temperature of the wall grows.

The example above shows that for the free surface flow problems characterized by temperature changes, the present fluid solver may be advantageous as it allows to easily account for temperature-dependent viscosity by simply adding a diffusion solver to an efficient semi-explicit mechanical solver.

\section{Summary and conclusion}

In this paper, a semi-explicit incompressible flow model was coupled to a heat equation solver. Being fully Lagrangian, the model automatically resolves convection and the heat mod- ule solves only the transient diffusion equation. The model belongs to the second generation of the Particle Finite Element Method solvers, where the particles-nodes are moved explicitly prior to the solution of the implicit problem. The solver for the thermally coupled flows presented here inherits the advantages of the mechanical solver formerly proposed by the authors in [22], namely, the possibility of using constant time step.

The thermal coupling was validated using several benchmark cases. The validation tests revealed that the proposed solver provides reliable solutions. Being semi-explicit the solver provides an attractive alternative to the fully implicit models, provided that the time step estimates are favorable. In particular, since in the mechanical part of the solver only the pressure is treated implicitly, favorable time step can be encountered in problems characterized by low viscosity values. For the flows characterized by high viscosity, the pro- 
posed solver is not advantageous (e.g., the casting problems, or melting problems where material viscosity at low temperatures is typically very high). This occurs due to the time step size restriction introduced by the explicit treatment of the viscous term. However, it is beneficial for thermo-mechanical fluid flow problems characterized by temperature-dependent viscosity.

\section{Compliance with ethical standards}

Conflicts of interest The authors declare that they have no conflict of interest.

\section{References}

1. ANSYS-Polyflow (2019) http://www.ansys.com/products/fluids/ ansys-polyflow

2. Arpaci V, Larsen P (1984) Convection heat transfer. Prentice Hall, New York

3. Aubry R, Idelsohn S, Oñate E (2005) Particle finite element method in fluid-mechanics including thermal convection-diffusion. Comput Struct 83(17):1459-1475 Advances in Meshfree Methods

4. Chorin A (1967) A numerical method for solving incompressible viscous problems. J Comput Phys 2:12-26

5. Codina R (2001) A stabilized finite element method for generalized stationary incompressible flows. Comput Methods Appl Mech Eng 190(20-21):2681-2706

6. Corzo S, Mrquez S, Ramajo D, Nigro N (2011) Numerical simulation of natural convection phenomena. ENIEF, Rosario, Argentina

7. Dadvand P, Rossi R, Oñate E (2010) An object-oriented environment for developing finite element codes for multi-disciplinary applications. Arch Comput Methods Eng 17(3):253-297

8. Davis G (1983) Natural convection of air in a square cavity: a benchmark numerical solution. Int J Numer Methods Fluids 3:249264

9. Dixit H, Babu V (2006) Simulation of high Rayleigh number natural convection in a square cavity using the lattice Boltzmann method. Int J Heat Mass Transf 49(3):727-739

10. Engineering-toolbox (2019) http://www.engineeringtoolbox.com/ water-dynamic-kinematic-viscosity-d-596.html

11. Franci A (2016) Unified lagrangian formulation for fluid and solid mechanics, fluid-structure interaction and coupled thermal problems using the PFEM. Springer, Cham (isbn 978-3319833415)

12. Friki A (1993) Simulation numerique 3D, en collocation Chebyshev, de phenomenes thermoconvectifs en centrifugation. These de 1'Universite Paris-Sud XI-Orsay

13. Hyre M (2002) Numerical simulation of glass forming and conditioning. J Am Ceram Soc 85(5):1047-1056

14. Idelsohn S, Marti J, Becker P, Oñate E (2014) Analysis of multifluid flows with large time steps using the particle finite element method. Int J Numer Methods Fluids 75(9):621-644

15. Idelsohn S, Marti J, Limache A, Oñate E (2008) Unified Lagrangian formulation for elastic solids and incompressible fluids. Application to fluid-structure interaction problems via the PFEM. Comput Methods Appl Mech Eng 197:1762-1776

16. Idelsohn S, Mier-Torrecilla M, Oñate E (2009) Multi-fluid flows with the particle finite element method. Comput Methods Appl Mech Eng 198(33-36):2750-2767

17. Idelsohn S, Mier-Torrecilla MD, Marti J, Oñate E (2011) The particle finite element method for multi-fluid flows. In: Oñate E, Owen R (eds) Particle-based methods. Springer, Dordrecht, pp 135-158
18. Idelsohn S, Oñate E, Del Pin F (2004) The particle finite element method: a powerful tool to solve incompressible flows with freesurfaces and breaking waves. Int J Numer Methods Eng 61:964989

19. Janssen R, Henkes R, Hoogendoorn C (1993) Transition to timeperiodicity of a natural-convection flow in 3D differentially heated cavity. Int J Heat Mass Transf 36:2927-2940

20. Marti J (2018) Exploration of kratos thermo-fluid capabilities for conjugate heat transfer problems. Technical report, CIMNE

21. Marti J, Idelsohn S, Oñate E (2018) A finite element model for the simulation of the ul-94 burning test. Fire Technol 54(6):1783-1805

22. Marti J, Ryzhakov P (2019) An explicit-implicit finite element model for the numerical solution of incompressible Navier-Stokes equations on moving grids. Comput Methods Appl Mech Eng (submitted)

23. Marti J, Ryzhakov P, Idelsohn S, Oñate E (2010) Combined Eulerian-PFEM approach for analysis of polymers in fire situations. Int J Numer Methods Eng 92:782-801

24. Mier Torrecilla M (2010) Numerical simulation of multi-fluid flows with the particle finite element method. Ph.D. Thesis, Universitat Politècnica de Catalunya

25. Muttin F, Coupez T, Bellet M, Chenot J (1993) Lagrangian finiteelement analysis of time-dependent viscous free-surface flow using an automatic remeshing technique: application to metal casting flow. Int J Numer Methods Eng 36(12):2001-2015

26. Nikulin D (1982) Applicability of the Boussinesq approximation for the solution of problems of unsteady natural concentration convection. Fluid Dyn 17(5):779-781

27. Oñate E, Idelsohn S, Del Pin F, Aubry R (2004) The particle finite element method: an overview. Int J Comput Methods 1:267-307

28. Oñate E, Marti J, Ryzhakov P, Rossi R, Idelsohn S (2013) Analysis of the melting, burning and flame spread of polymers with the particle finite element method. Comput Assist Methods Eng Sci $20: 165-184$

29. Oñate E, Rojek J, Chiumenti M, Idelsohn S, Del Pin F, Aubry R (2006) Advances in stabilized finite element and particle methods for bulk forming processes. Comput Methods Appl Mech Eng 195(48-49):6750-6777

30. Radovitzky R, Ortiz M (1998) Lagrangian finite element analysis of Newtonian fluid flows. Int J Numer Methods Eng 43(4):607-619

31. Ramsak M (2015) Conjugate heat transfer of backward-facing step flow: a benchmark problem revisited. Int J Heat Mass Transf 84:791-799

32. Ramaswamy B, Kawahara M (1987) Lagrangian finite element analysis applied to viscous free surface fluid flow. Int J Numer Methods Fluids 7(9):953-984

33. Ramaswamy B, Kawahara M, Nakayama T (1986) Lagrangian finite element method for the analysis of two-dimensional sloshing problems. Int J Numer Methods Fluids 6(9):659-670

34. Ryzhakov P (2017) A modified fractional step method for fluidstructure interaction problems. Rev Int de Métod Numér para Cálc y Diseño en Ing 33(1-2):58-64

35. Ryzhakov P, Garcia J, Oñate E (2016) Lagrangian finite element model for the 3D simulation of glass forming processes. Comput Struct 177:126-140

36. Ryzhakov P, Marti J (2018) A semi-explicit multi-step method for solving incompressible Navier-Stokes equations. Appl Sci $8(1): 119$

37. Ryzhakov P, Marti J, Idelsohn S, Oñate E (2017) Fast fluid-structure interaction simulations using a displacement-based finite element model equipped with an explicit streamline integration prediction. Comput Methods Appl Mech Eng 315:1080-1097

38. Ryzhakov P, Oñate E (2017) A finite element model for fluidstructure interaction problems involving closed membranes, internal and external fluids. Comput Methods Appl Mech Eng 326:422445 
39. Ryzhakov P, Rossi R, Oñate E (2012) An algorithm for the simulation of thermally coupled low speed flow problems. Int J Numer Methods Fluids 70(1):1-19

40. Ryzhakov PB (2017) An axisymmetric PFEM formulation for bottle forming simulation. Comput Part Mech 4(1):3-12

41. Sklar D, Gimenez J, Nigro N, Idelsohn S (2012) Thermal coupling in particle finite element method-second generation. Mec Comput 31:4143-4152
42. Zhu M, Scott MH (2017) Unified fractional step method for Lagrangian analysis of quasi-incompressible fluid and nonlinear structure interaction using the PFEM. Int J Numer Methods Eng 109(9):1219-1236

Publisher's Note Springer Nature remains neutral with regard to jurisdictional claims in published maps and institutional affiliations. 